\title{
Exciton annihilation in dye-sensitized nanocrystalline semiconductor films
}

\author{
Akihiro Namekawa, Ryuzi Katoh ${ }^{*}$ \\ Department of Chemical Biology and Applied Chemistry, College of Engineering, Nihon \\ University, Koriyama, Fukushima 963-8642, Japan \\ "Corresponding author. \\ E-mail address: rkatoh@chem.ce.nihon-u.ac.jp (R. Katoh)
}

\begin{abstract}
Exciton annihilation in dye-sensitizednanocrystalline semiconductor $\left(\mathrm{Al}_{2} \mathrm{O}_{3}\right)$ films has been studied through laser-induced fluorescence spectroscopy. The relative quantum yield of the fluorescence decreases with increasing excitation light intensity, the indication being that exciton annihilation occurred. The rate constants of the annihilation were estimated for three dyes, N719, D149, and MK2, that are known to be sensitizing dyes for efficient dye-sensitized solar cells. The hopping time between dye molecules and the diffusion length of excitons within their lifetime were also estimated to facilitate discussion of the relevance of exciton annihilation to primary processes in dye-sensitized solar cells.
\end{abstract}

\section{Introduction}

Dye-sensitized solar cells (DSSCs) have been extensively studied as an attractive new class of solar cells [1-3]. It has been argued that DSSCs have several advantages, including low cost, flexibility, color availability, and potential for indoor applications, compared with other types of solar cells, such as Si-based solar cells [1-3]. Although DSSCs have potential utility inpractical applications, they have not yet been used in commercial products, because their device performanceand long-term stability have not yet been optimized. To date, the official record for solar-light-to-energy conversion efficiency $(\eta)$ ofDSSCs is $11.9 \%[4]$. The primary processes that occur inDSSCs have been extensively studiedin an effort to improve device performance [5-9].Nanocrystalline semiconductor films, which are prepared by sintering of semiconductor nano-particles, are one of the most important components of DSSCs. Sensitizing dyes are chemically adsorbed onto the surface of the particles, and the product is a nano-porous, dye-sensitized film. The dyes are attached directly onto the surface, and therefore electron injection from optically excited dyes to the semiconductor particles can occur efficiently if the edge level of the conduction band is lower than the level of the lowest unoccupied molecular orbital (LUMO) of the dye

(C) 2016. This manuscript version is made available under the Elsevier user license

http://www.elsevier.com/open-access/userlicense/1.0/ 
[8]. After electron injection, the dyes are oxidized, and they are immediately reduced by a redox mediator in the solution that penetrates the nano-pore structure of the films. This unique mechanismis the key technology that enables the high performance of DSSCs to be realized.

Recently, migration of intermediates produced by photo-absorption generated on nanocrystalline films has been noted. Oxidized forms of dyes (holes) have been found to migrate on the surface of nano-particles [10-12]. Recently, formation of a singlet excited state by fusion of two triplet excited states after migration on the surface, a process called up-conversion, has been studied [13]. Accordingly, the effect of the migration of intermediate species on the performance of DSSCs should be clarified forfurther improvement of the devices.

Here we studied exciton migration in dye-sensitized nanocrystalline semiconductor films. Electron injection from excited dyes to $\mathrm{Al}_{2} \mathrm{O}_{3}$ films is inhibited because of the high position of the conduction band edge. We therefore used $\mathrm{Al}_{2} \mathrm{O}_{3}$ films instead of $\mathrm{TiO}_{2}$ films which are widely used in DSSCs, to study exciton migration. The molecular structures of the dyes used in this study (N719, D149, and MK2) are shown in Fig. 1.It is known that the performance of DSSCs based on N719 [14], D149 [15], and MK2[16] is relatively high. To study exciton migration, we observed exciton annihilation, in which two excitons ( $\left.\mathrm{M}^{*}\right)$ become one excitonas the result of a collision $\left(M^{*}+M^{*} \rightarrow M^{*}\right)$. Actually, we observed a decrease of relative fluorescence quantum yield at high excitation light intensities. We were able to estimate the rate constant of exciton annihilation and using that rate constant, we also estimated the hopping time between two dye molecules and the diffusion length of an exciton within its lifetime based on diffusion theory.

\section{Experimental}

$\mathrm{Al}_{2} \mathrm{O}_{3}$ films were prepared by spin-coating an $\mathrm{Al}_{2} \mathrm{O}_{3}$ slurry (NanoTek, ALAP15WT\%-X480) ontoquartz slides $(2.5 \mathrm{~cm} \times 2.5 \mathrm{~cm})$. The diameter of the particles in the slurry was $31 \mathrm{~nm}$. After calcination for $1 \mathrm{~h}$ at $800 \mathrm{~K}$, we obtained films that were about $1 \mu \mathrm{m}$ thick. N719 (Sigma-Aldrich), D149 (Sigma-Aldrich)and MK2 [17] were dissolved in acetonitrile : tert-buthylalcohol (1:1)(1 mM), ethanol $(1 \mathrm{mM})$ and toluene $(0.35 \mathrm{mM})$, respectively. The nanocrystalline $\mathrm{Al}_{2} \mathrm{O}_{3}$ films were immersed in the dye solutions for $20 \mathrm{~h}$, so that the dye could adsorb onto the semiconductor surface. The films then were rinsed with ethanol to remove physisorbed dyes and dried in air.

Absorption and luminescence spectra of the films were measured with an absorption spectrometer (V-670, JASCO) and fluorescence spectrometer (RF-5300PC, Shimadzu), respectively.Luminescence decay curves were obtained by time-correlated, single-photon 
counting. The sample was excited by the second harmonic $(400 \mathrm{~nm})$ of a Ti:sapphire laser (Spectra-Physics, Tsunami).Repetition rate of the oscillator $(80 \mathrm{MHz})$ was reduced to $8 \mathrm{MHz}$ with a pulse selector (Spectra-Physics, Model 3980). Intensity of the laser was less than $10^{-9}$ $\mathrm{Jcm}^{-2}$. Luminescence was detected with a photomultiplier tube (Hamamatsu, E3809U-50) after being dispersed with a monochromator (Jovin Yvon, H20). The dependence of the luminescence measurements on excitation light intensity was studied with a $\mathrm{Nd}^{3+}:$ YAGlaser (10 ns pulse duration,HOYA Continuum, Surelite II). The second harmonic (532 nm) of the laser was used for excitation.Spot size of the excitation laser was $6 \mathrm{~mm}$ in diameter. Luminescence was detected with a photomultiplier tube (Hamamatsu, R928) after being dispersed with a monochromator (Ritsu, MC-100G). Luminescence spectra under pulsed laser excitation condition could be also recorded. The intensity of the laser pulse was varied with calibrated neutral density filters and absolute value was measured with a pyroelectric energy meter (Ophir, PE25-C).All measurements were carried out at room temperature.

\section{Results and discussion}

To determine the number of dye molecules adsorbed onto the films, dye molecules were rinsed away with alkali water. Figure 2 shows the absorbance $A_{\text {obs }}$ of the rinse solutions of thefilmsas a function of the concentration of the N719 dyesolutions, [N719] $]_{\text {sol }}$, used for the preparation of the dye-sensitized films. That $A_{\text {obs }}$ tended to saturate at relatively high dye concentrations wasindicative ofLangmuir-type adsorption. In such a case, the coverageof dye on the surface can be expressed as $A_{\text {obs }} / A_{\text {full, }}$ where $A_{\text {full }}$ is the absorbance at full monolayer coverage on the surface(saturation value). Accordingly, the Langmuir-type isotherm canbe expressed as

$$
\frac{A_{\mathrm{obs}}}{A_{\text {full }}}=\frac{K_{L}[N 719]_{\mathrm{sol}}}{1+K_{L}[N 719]_{\mathrm{sol}}}
$$

where $K_{\mathrm{L}}$ is the adsorption coefficient $\left(\mathrm{M}^{-1}\right)$. As shown in Fig. 2, the solid line generated from Eq. (1) using the values of $A_{\text {full }}=0.26$ and $K_{\mathrm{L}}=12 \mathrm{mM}^{-1}$ could reproduce the experimental data. For DSC devices, densely adsorbed films have generally been used to realize high light harvesting efficiency and therefore the film prepared from $1 \mathrm{mM}$ dye solution, which resulted in nearly full coverage, was used for spectroscopic measurements in the present study. The number of dye molecules, $N_{\text {ad }}{ }^{\text {dye }}$, adsorbed onto the film could also be estimatedfrom $A_{\text {obs }}$ and was found to be $N_{\text {ad }}$ dye $=5 \times 10^{16}$ molecules. For other dye systems, full coverage films were used; $N_{\text {ad }}{ }^{\text {dye }}$ was estimated to be $N_{a d}{ }^{\text {dye }}=9 \times 10^{16}$ molecules for D149 
films and $6 \times 10^{16}$ molecules for MK2 films. Absorption and luminescence spectra normalized to the highest absorption and luminescence of the films are shown in Fig. 3. Note that the luminescence observed can be assigned to phosphorescence for the N719 film and to fluorescence for the D149 and MK2 films.For D149, luminescence spectra were recorded at different excitation laser fluences, $F_{\mathrm{ex}}=7 \mathrm{mJcm}^{-2}$ (solid line), $F_{\mathrm{ex}}=0.11 \mathrm{mJcm}^{-2}$ (solid circles) and $F_{\mathrm{ex}}=0.003 \mathrm{mJcm}^{-2}$ (open circles). It is obvious that the spectral shape is not sensitive to laser fluence.

Figure 4 showsluminescence decay profiles obtained with 400-nm laser pulse excitation. Becausethe excitation intensity wastoo weak $\left(<10^{-9} \mathrm{Jcm}^{-2}\right)$, exciton annihilation wasnegligible in this case. The observed luminescence wavelengths for N719, D149 and MK2 were $750 \mathrm{~nm}, 670 \mathrm{~nm}$ and $700 \mathrm{~nm}$, respectively. The decay curve could be fitted with a double exponential function $\left[A_{1} \exp \left(1 / \tau_{1}\right)+A_{2} \exp \left(1 / \tau_{2}\right)\right]$, where $A$ is amplitude and $\tau$ is lifetime. The fitting results for each of the curves, including fitting parameters, are shown in Fig. 4. Average lifetimes, the values of $\tau_{\text {ave }}\left(A_{1} \times \tau_{1}+A_{2} \times \tau_{2}\right)$, are used in the later discussion. They were calculated to be $17 \mathrm{~ns}$ for N719,0.28 ns for D149 and 0.33 ns for MK2 films.

Figure 5 shows luminescence intensity, $l_{\text {lumi }}$, as a function of excitation laser fluence, $F_{\text {ex }}$ $\left(\mathrm{Jcm}^{-2}\right)$, obtained with 532-nm laser pulse excitation. Observed wavelengths for N719, D149 and MK2 were $750 \mathrm{~nm}, 700 \mathrm{~nm}$ and $700 \mathrm{~nm}$, respectively. It is obvious that lumiWas proportional to $F_{\text {ex }}$ at low $F_{\text {ex }}$ values and tended to saturate with increasing $F_{\text {ex }}$. Finally, lumiwas proportional to $F_{\mathrm{ex}}{ }^{0.5}$. We confirm that this is not due to photo-decomposition of dyes from repeated measurements. Accordingly, this result clearly indicated that exciton annihilation occurred under high-density excitation conditions.

Exciton annihilation is a disproportionation reaction of exciton $\mathrm{M}^{*}$ described by $\mathrm{M}^{*}+\mathrm{M}^{*} \rightarrow$ $\mathrm{M}^{*}$ ). Such a process can be characterized by the following rate equation:

$$
\frac{d\left[M^{*}\right]}{d t}=\alpha I_{\mathrm{ex}}-\frac{1}{\tau_{\mathrm{ave}}}\left[M^{*}\right]-\gamma_{\mathrm{EX}}\left[M^{*}\right]^{2}
$$

where $\left[\mathrm{M}^{*}\right]$ is the concentration of excitons, $\alpha$ is the absorption coefficient, $l_{\mathrm{ex}}$ isthe excitation light intensity (photons $\mathrm{cm}^{-2} \mathrm{~s}^{-1}$ ) and $\gamma_{\mathrm{Ex}}$ is the rate constant of exciton annihilation. Because $\left[\mathrm{M}^{*}\right]$ is proportional to $I_{\text {lumi, }} \gamma_{\mathrm{EX}}$ can be evaluated from luminescence measurements if $\alpha$ and $\tau_{\text {ave }}$ are known. As shown in Fig. 5 , the dependence of/lumi on $F_{\text {ex }}$ changed from $F_{\text {ex }}{ }^{1.0}$ to $F_{\mathrm{ex}}{ }^{0.5}$ at the critical excitation laser fluence, $F_{\mathrm{ex}}{ }^{\mathrm{c}} \cdot \mathrm{In}$ the present results, critical excitation light intensity $l_{\mathrm{ex}}{ }^{\mathrm{c}}$ values could be estimated from $F_{\mathrm{ex}}{ }^{\mathrm{c}}$ to be $1.5 \times 10^{23} \mathrm{~cm}^{-2} \mathrm{~s}^{-1}$ for $\mathrm{N} 719,5.0 \times 10^{22}$ 
$\mathrm{cm}^{-2} \mathrm{~s}^{-1}$ for D149 and1.5 $\times 10^{22} \mathrm{~cm}^{-2} \mathrm{~s}^{-1}$ for MK2 using a value of $10 \mathrm{~ns}$ for the laser pulse duration. Under steady state conditions, $\mathrm{d}\left[\mathrm{M}^{*}\right] / \mathrm{dt}=0$ and $\gamma_{\mathrm{Ex}}$ can be expressed using $l_{\mathrm{ex}}{ }^{\mathrm{C}}$ as

$\gamma_{\mathrm{EX}}=\frac{1}{\alpha \tau_{\mathrm{ave}}{ }^{2} I_{\mathrm{eX}}^{\mathrm{c}}}$

To estimate $\alpha$ values, heterogeneity of the film should be taken into account. Excitation light can only be absorbed by dyes adsorbed onto the surface of semiconductor nanoparticles. In other words, excitons are concentrated in the dyeregion of the film and there are no excitons in the semiconductor region. Thus, the effective absorption coefficient can be evaluated by reducing the thickness of the films. Actually, the thickness of the dye layer can be estimated from the number of dye molecules adsorbed onto the surface if the volume occupied by a single dye molecule, $V_{\text {dye }}$, isknown. We assumed $V_{\text {dye }}$ to be $V_{\text {dye }}=1$ $\mathrm{nm}^{3}$ based on the area occupied by a single $\mathrm{N} 719$ dye molecule on the $\mathrm{TiO}_{2}$ surface obtained from the adsorption isotherm [18] and the atomic force microscopy image of N719 on $\mathrm{TiO}_{2}$ single crystals [19]. The same $V_{\text {dye }}$ value was used for the other dyes because the number of adsorbed D149 and MK2 dye molecules was similar to the number of adsorbed N719 molecules, as mentioned above. Accordingly, based on the absorbance of the films, theeffective values of $\alpha$ could be estimated to be $\alpha=2.5 \times 10^{4} \mathrm{~cm}^{-1}$ for N719, $\alpha=1.5 \times 10^{5}$ $\mathrm{cm}^{-1}$ for D149 and $\alpha=6.5 \times 10^{4} \mathrm{~cm}^{-1}$ for MK2 at $532 \mathrm{~nm}$. Based on the foregoing considerations, the value of $\gamma_{E x}$ Could be estimated to be $\gamma_{E X}=1 \times 10^{-12} \mathrm{~cm}^{3} \mathrm{~s}^{-1}$ for N719, $\gamma_{\mathrm{EX}}$ $=2 \times 10^{-9} \mathrm{~cm}^{3} \mathrm{~s}^{-1}$ for D149 and $\gamma_{\mathrm{EX}}=1 \times 10^{-8} \mathrm{~cm}^{3} \mathrm{~s}^{-1}$ for MK2 .

Exciton annihilation rate constantsof singlet excitonsin single crystals havebeen studied in aromatic hydrocarbon crystals. For example, $\gamma_{\mathrm{Ex}}$ values for single crystals were determined to be $\gamma_{\mathrm{EX}}=1.7 \times 10^{-9} \mathrm{~cm}^{3} \mathrm{~s}^{-1}$ for naphthalene[20], $\gamma_{\mathrm{EX}}=1 \times 10^{-8} \mathrm{~cm}^{3} \mathrm{~s}^{-1}$ for anthracene[20] and $\gamma_{\mathrm{EX}}=1 \times 10^{-8} \mathrm{~cm}^{3} \mathrm{~s}^{-1}$ for the free exciton in $\beta$-perylene [21].These rate constants tend to increase with increasing molecular size. This trend suggests thatintermolecular interactions play an important roleinexciton hopping between molecules. For Rucomplexes, intersystem crossing (ISC) from the Frank-Condon singlet state is known to be extremely fast, for example, a 100-fs decay time of the singletexcited state through ISC has been observed for the N719 dye in solution [22]. Accordingly, exciton annihilation in Ru-complex crystals has been observed only for triplet excitons. The exciton annihilation rate constant, $\gamma_{\mathrm{EX}}$, of triplet excitons in $\mathrm{Ru}\left[(\mathrm{bpy})_{3}\right] \mathrm{X}_{2}\left(\mathrm{X}=\mathrm{ClO}_{4}{ }^{-}\right.$and $\left.\mathrm{PF}_{6}{ }^{-}\right)$crystals has been determinedto be $\gamma_{\mathrm{Ex}}=6.2 \mathrm{x}$ $10^{-12} \mathrm{~cm}^{3} \mathrm{~s}^{-1}$ for $\mathrm{Ru}\left[(\mathrm{bpy})_{3}\right]\left(\mathrm{ClO}_{4}^{-}\right)_{2}$ and $\gamma_{\mathrm{EX}}=1.8 \times 10^{-12} \mathrm{~cm}^{3} \mathrm{~s}^{-1}$ for $\mathrm{Ru}\left[(\mathrm{bpy})_{3}\right]\left(\mathrm{PF}_{6}{ }^{-}\right)_{2}[23]$. The rate constant increaseswith decreasing size of the counter anion. This trend again suggests 
the important role of intermolecular interactionsin determiningthe rate of exciton hopping

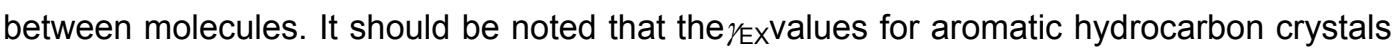
are much larger than those of Ru-complexes. This difference is due to the difference of the spin multiplicity of the excitons, namely a singlet exciton for aromatic hydrocarbon crystals and a triplet for Ru-complexes.In fact, the $\gamma_{\text {Ex }}$ for triplet excitons in aromatic hydrocarbon crystalshas been found to be $\gamma_{\mathrm{Ex}}=1.7 \times 10^{-11} \mathrm{~cm}^{3} \mathrm{~s}^{-1}$ for anthracene [20].

We have compared the exciton annihilation rate constants obtained in the present study with those reported previously. For $\mathrm{N} 719$ on $\mathrm{Al}_{2} \mathrm{O}_{3}$ films, the $\gamma_{\mathrm{EX}}\left(1 \times 10^{-12} \mathrm{~cm}^{3} \mathrm{~s}^{-1}\right)$ was similar to that for $\operatorname{Ru}\left[(\mathrm{bpy})_{3}\right]$ crystals. This similarity suggests that the intermolecular distance for $\mathrm{N} 719$ on the film is similar to that of $\mathrm{Ru}\left[(\mathrm{bpy})_{3}\right]$. For D149and MK2 on $\mathrm{Al}_{2} \mathrm{O}_{3}$ films, we compared the $\gamma_{\text {EX }}$ values with those of aromatic crystals because $\pi$-electron

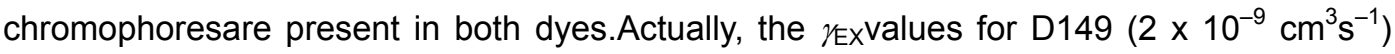
and MK2 $\left(1 \times 10^{-8} \mathrm{~cm}^{3} \mathrm{~s}^{-1}\right)$ on $\mathrm{Al}_{2} \mathrm{O}_{3}$ films were similar to those of aromatic hydrocarbon crystals. These results suggest that the intermolecular interactions of dyes adsorbed on the $\mathrm{Al}_{2} \mathrm{O}_{3}$ films are similar to those in crystals.

If the annihilation reaction is diffusion limited, a diffusion constant, $D$, can be calculated from the exciton annihilation constant; in that case $D=\gamma_{\mathrm{EX}} /(4 \pi R)$, where $R$ is the reaction radius, which is commonly estimated from the cross sectional area of the size of the molecule. Accordingly, $R=1 \mathrm{~nm}$ has been used as an acceptable value for rough estimation purposes. Based on diffusion theory, the hopping time, $\tau_{\text {hop }}$, of excitons between molecules can be evaluated using the relationship $\tau_{\text {hop }}=d_{\text {site }}{ }^{2} /(2 D)=\left(4 \pi R d_{\text {site }}{ }^{2}\right) /\left(2 \gamma_{\text {EX }}\right)$, where $d_{\text {site }}$ is the intermolecular distance.Accordingly, the value of $\tau_{\text {hop }}$ can be estimated to be $\tau_{\text {hop }}=1 \mathrm{x}$ $10^{-8} \mathrm{~s}$ for N719, $4 \times 10^{-12} \mathrm{~s}$ for D149 and $1 \times 10^{-12} \mathrm{~s}$ for MK2.Using these values and the exciton lifetime, $\tau_{\text {ave }}$, the diffusion distance, $L_{\mathrm{d}}$, could be estimated based on the relationship $L_{d}=\left(D \tau_{\text {ave }}\right)^{1 / 2}=\left(\gamma_{\mathrm{Ex}} \tau_{\text {ave }} / 4 \pi R\right)^{1 / 2}$. Accordingly, the $L_{d}$ value could be estimated to be $0.5 \mathrm{~nm}$ for N719, $5 \mathrm{~nm}$ for D149 and10 $\mathrm{nm}$ for MK2.

Consideringthe foregoing discussion, we examined the relationship between exciton migration and electron injection from excited dye molecules to the semiconductor. Note that experimental condition in the present study is different from that in electron injection studies. Actually, $\mathrm{Al}_{2} \mathrm{O}_{3}$ was used instead of $\mathrm{TiO}_{2}$ and all experiments were carried out under ambient air. For N719, many studies on electron injection processes have been carried out to elucidate the mechanism $[5,6,8,9,25]$. Although there have been many debatable hypotheses concerning these processes, electron injection occurs stepwise; ultrafast $(<200$ fs) and slow (10-500 ps) injection processes have been observed simultaneously. It should be noted that the rate of electron injection is effectively reduced when dye-sensitized $\mathrm{TiO}_{2}$ films are immersed into electrolyte, although details of the mechanism are unknown 
$[6,8,9,25]$. As estimated above, the hopping time, $\tau_{\text {hop }}$, of the triplet exciton in N719 films is estimated to be $1 \times 10^{-8}$ s. Electron injection from $\mathrm{N} 719$ to $\mathrm{TiO}_{2}$ could therefore occur before exciton hopping, even in an electrolyte. For D149, electron injection dynamics have been studied based on femtosecond transient absorption measurements [25] and ultrafast $(<100$ fs) and slow (10-50 ps) injection processes have been observed. These processes are slightly faster than those observed for N719 [6,8,9,25]. Accordingly, the electron injection process can compete with exciton hopping; in particular, electron injection after exciton migration is possible. For MK2, only an ultrafast electron injection process in dried film has been observed [26]. In such a case, electron injection occurs before exciton hopping to other molecules.

In conclusion, we observed exciton annihilation in dye-sensitized, nanocrystalline $\mathrm{Al}_{2} \mathrm{O}_{3}$ films. Exciton annihilation rate constants, $\gamma_{\mathrm{EX}}$, were estimated to be $1 \times 10^{-12} \mathrm{~cm}^{3} \mathrm{~s}^{-1}$ for N719, $2 \times 10^{-9} \mathrm{~cm}^{3} \mathrm{~s}^{-1}$ for D149 and $1 \times 10^{-8} \mathrm{~cm}^{3} \mathrm{~s}^{-1}$ for MK2. The hopping time and diffusion length of excitons were evaluated based on diffusion theory. Accordingly, we conclude that in some cases, the process of excitonhopping can compete with the electron-injection process in dye-sensitized solar cells.

\section{Acknowledgments}

We thank Dr. Nagatoshi Koumura for providing the MK2 sample.This work was supported by SENTAN, JST.

\section{References}

[1] A. Hagfeldt, M. Grätzel, Chem. Rev. 95 (1995) 49.

[2] A. Hagfeldt, G. Boschloo, L. Sun, L. Kloo, H. Pettersson, Chem. Rev. 110 (2010)6595.

[3] K. Kalyanasundaram, Dye-sensitized Solar Cells, EPFL Press, Lausanne, 2010.

[4] http://www.nrel.gov/ncpv/images/efficiency_chart.jpg

[5] J.B. Asbury, E. Hao, Y. Wang, H.N. Ghosh, T. Lian, J. Phys. Chem. B 105 (2001)4545.

[6] J.R. Durrant, S.A. Haque, E. Palomares, Chem. Commun. (2006) 3279.

[7] S. Ardo, G.J. Meyer, Chem. Soc. Rev. 38 (2009) 115.

[8] R. Katoh, A. Furube, J. Photochem. Photobiol. C20 (2014) 1.

[9] C. Martin, M. Ziółek, A. Douhal,J. Photochem. Photobiol. C26 (2016) 1.

[10] P. Bonhôte, E. Gogniat, S. Tingry, C. Barbé, N. Vlachopoulos, F. Lenzmann, P. Comte, M. Grätzel, J. Phys. Chem. B, 102 (1998) 1498.

[11] S. Ardo, G. J. Meyer, J. Am. Chem. Soc. 132 (2010) 9283.

[12] D. Moia,U. B. Cappel, T. Leijtens, X. Li,A. M. Telford,H. J. Snaith, B. C. O'Regan,J. Nelson, P. R. F. Barnes, J. Phys. Chem. C 119 (2015) 18975. 
[13]J. S. Lissau, D. Nauroozi, M.-P. Santoni,T. Edvinsson, S. Ott, J. M. Gardner, A. Morandeira, J. Phys. Chem. C 119 (2015) 4550.

[14] M.K. Nazeeruddin, F. De Angelis, S. Fantacci, A. Selloni, G. Viscardi, P. Liska, S. Ito, B. Takeru, M. Grätzel, J. Am. Chem. Soc. 127 (2005) 16835.

[15] T.Horiuchi,H.Miura,K.Sumioka,S.Uchida,J.Am.Chem.Soc.126(2004)12218.

[16] N. Koumura,Z.-S. Wang, S. Mori, M. Miyashita, E. Suzuki, K. Hara, J. Am. Chem. Soc. 128 (2006) 14256.

[17] Provided by Dr. Nagatoshi Koumura.

[18] R. Katoh, K. Yaguchi, A. Furube, Chem. Phys. Lett. 511 (2011) 336.

[19] A. Sasahara, C.L. Pang, H. Onishi, J. Phys. Chem. B 110 (2006) 4751.

[20] M. Pope and C. E. Swenberg, Electronic Processes in Organic Crystals and Polymers, Oxford University Press, New York, 1999.

[21] T. Yago, Y. Tamaki,A. Furube, R. Katoh, Phys. Chem. Chem. Phys. 10 (2008) 4435.

[22] A. C. Bhasikuttan, T. Okada, J. Phys. Chem. B, 108 (2004) 12629.

[23] N. Ikeda, A. Yoshimura, M. Tsushima, T. Ohno, J. Phys. Chem. A 104 (2000)6158.

[25] M. Juozapavicius,M. Kaucikas,J. J. van Thor, B. C. O’Regan, J. Phys. Chem. C 117 (2013) 116.

[26] Z.-S. Wang, N. Koumura, Y. Cui, M. Takahashi, H. Sekiguchi, A. Mori, T. Kubo, A. Furube, K. Hara, Chem. Mater. 20 (2008)3993. 
Figure captions

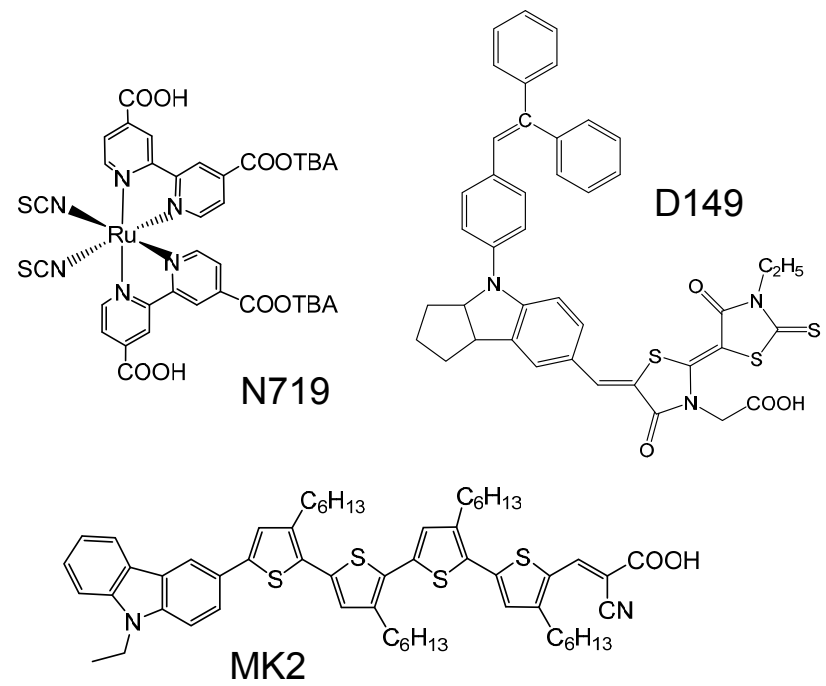

Fig. 1. Molecular structure of dyes studied. TBA in N719 represents tetrabutylammonium. 


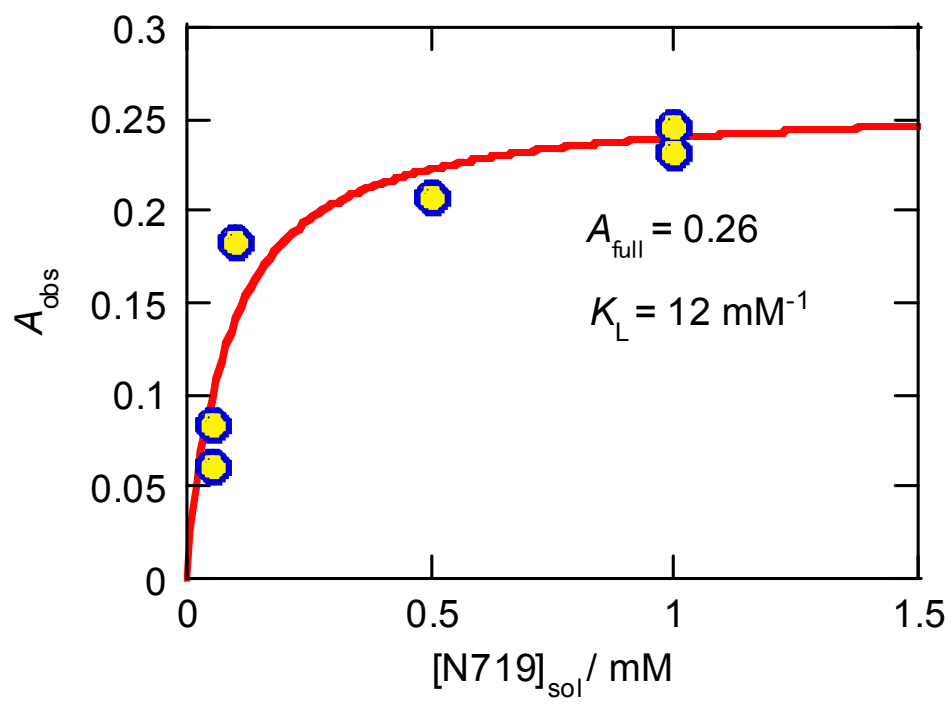

Fig. 2. Adsorption isotherm of $\mathrm{N} 719$ dye on nanocrystalline $\mathrm{Al}_{2} \mathrm{O}_{3}$ films. The solid line represents the Langmuir-type isotherm using the values of $A_{\text {full }}=0.26$ and $K_{\mathrm{L}}=12 \mathrm{mM}^{-1}$. 

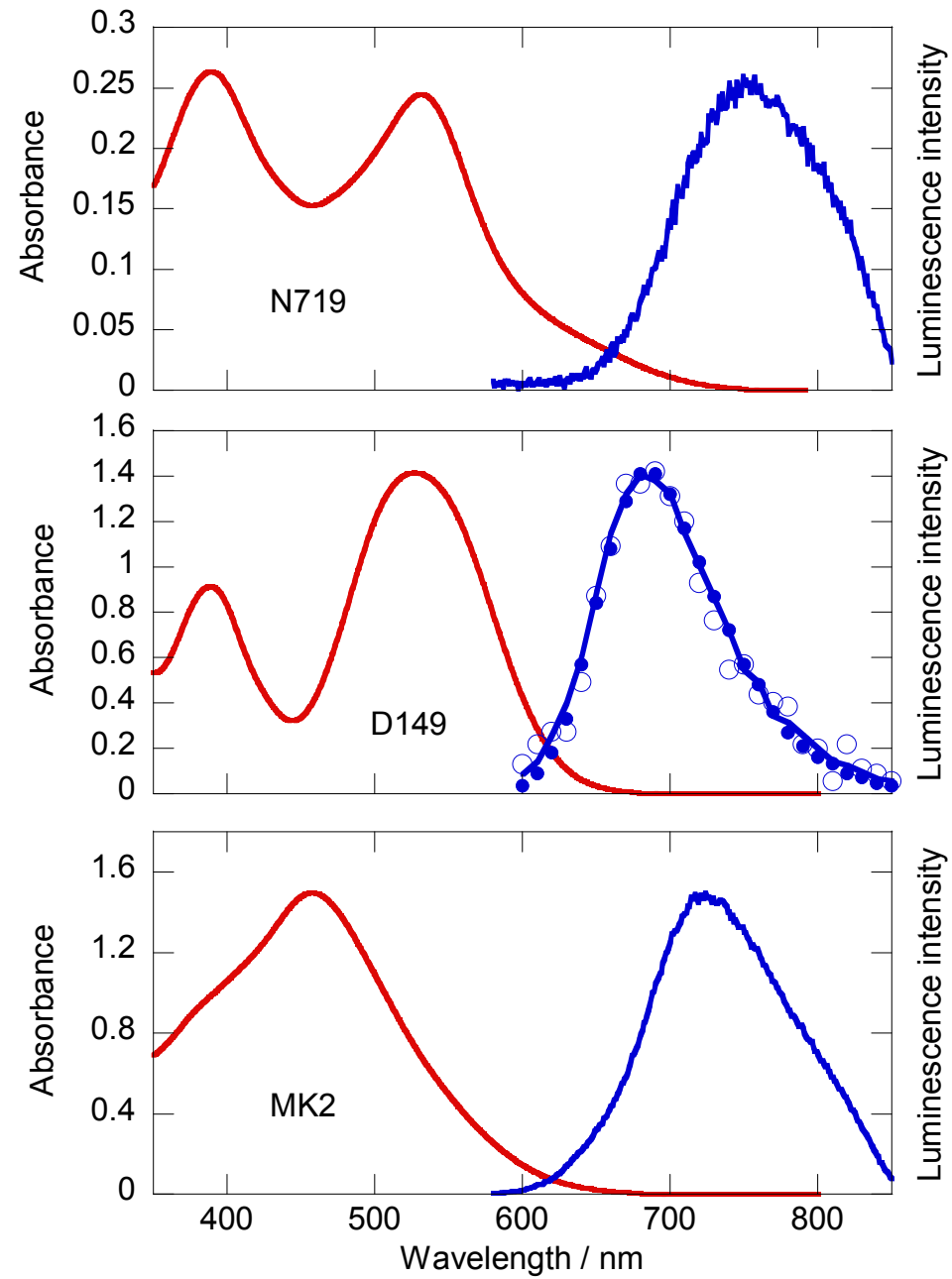

Fig. 3. Absorption and luminescence spectra of N719, D149, and MK2 adsorbed onto nanocrystalline $\mathrm{Al}_{2} \mathrm{O}_{3}$ films. For $\mathrm{D} 149$, luminescence spectra were recorded at different excitation laser fluences, $F_{\mathrm{ex}}=7 \mathrm{mJcm}^{-2}$ (solid line), $F_{\mathrm{ex}}=0.11 \mathrm{mJcm}^{-2}$ (solid circles) and $F_{\mathrm{ex}}$ $=0.003 \mathrm{mJcm}^{-2}$ (open circles). 

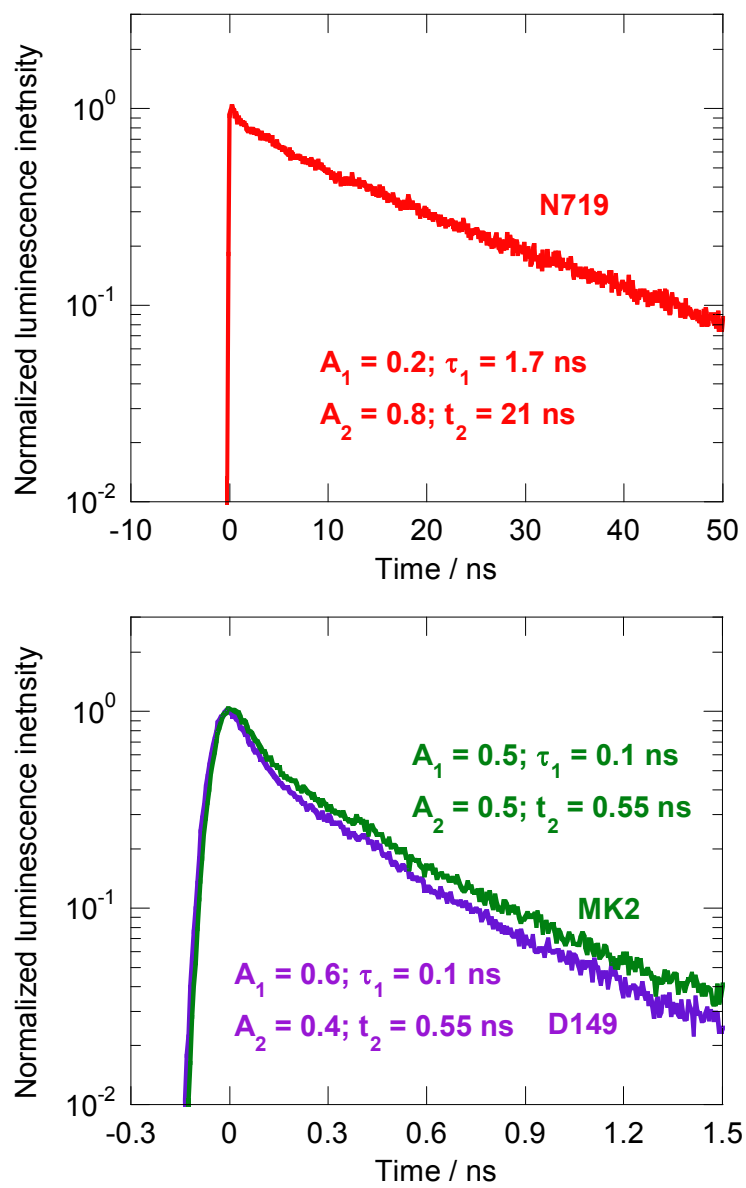

Fig. 4. Luminescence lifetime measurements for N719, D149, and MK2 adsorbed onto nanocrystalline $\mathrm{Al}_{2} \mathrm{O}_{3}$ films. The excitation wavelength was $400 \mathrm{~nm}$. Luminescence for $\mathrm{N} 719$, D149, and MK2 films was observed at $750 \mathrm{~nm}, 670 \mathrm{~nm}$, and $700 \mathrm{~nm}$, respectively. The fitting parameters shown in the panels correspond to the double exponential function $\left(A_{1} \exp \left(1 / \tau_{1}\right)+\right.$ $\left.A_{2} \exp \left(1 / \tau_{2}\right)\right)$. 


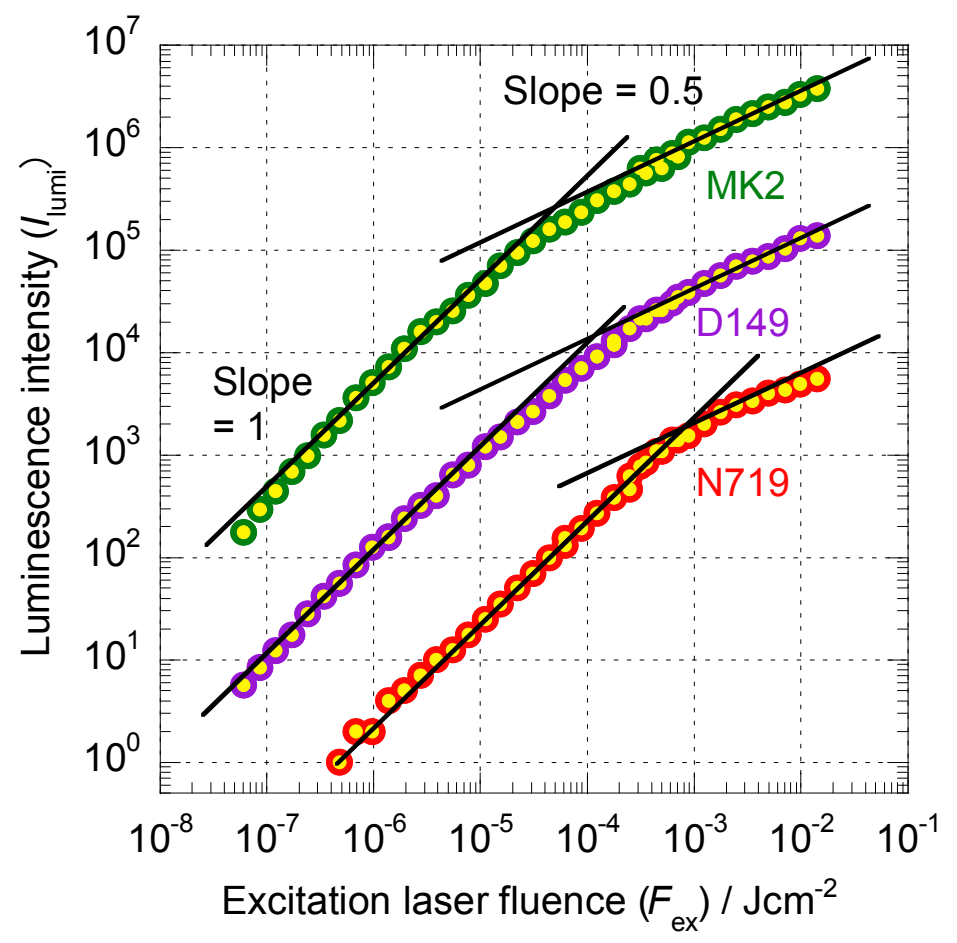

Fig. 5. Luminescence intensity as a function of excitation laser fluence for N719, D149, and MK2 adsorbed onto nanocrystalline $\mathrm{Al}_{2} \mathrm{O}_{3}$ films. The excitation wavelength was $532 \mathrm{~nm}$. Luminescence for N719, D149, and MK2 films was observed at $750 \mathrm{~nm}, 700 \mathrm{~nm}$, and 700 $\mathrm{nm}$, respectively. Straight lines fit to data have slopes of 1 or 0.5 , as indicated. 
Graphical Abstract

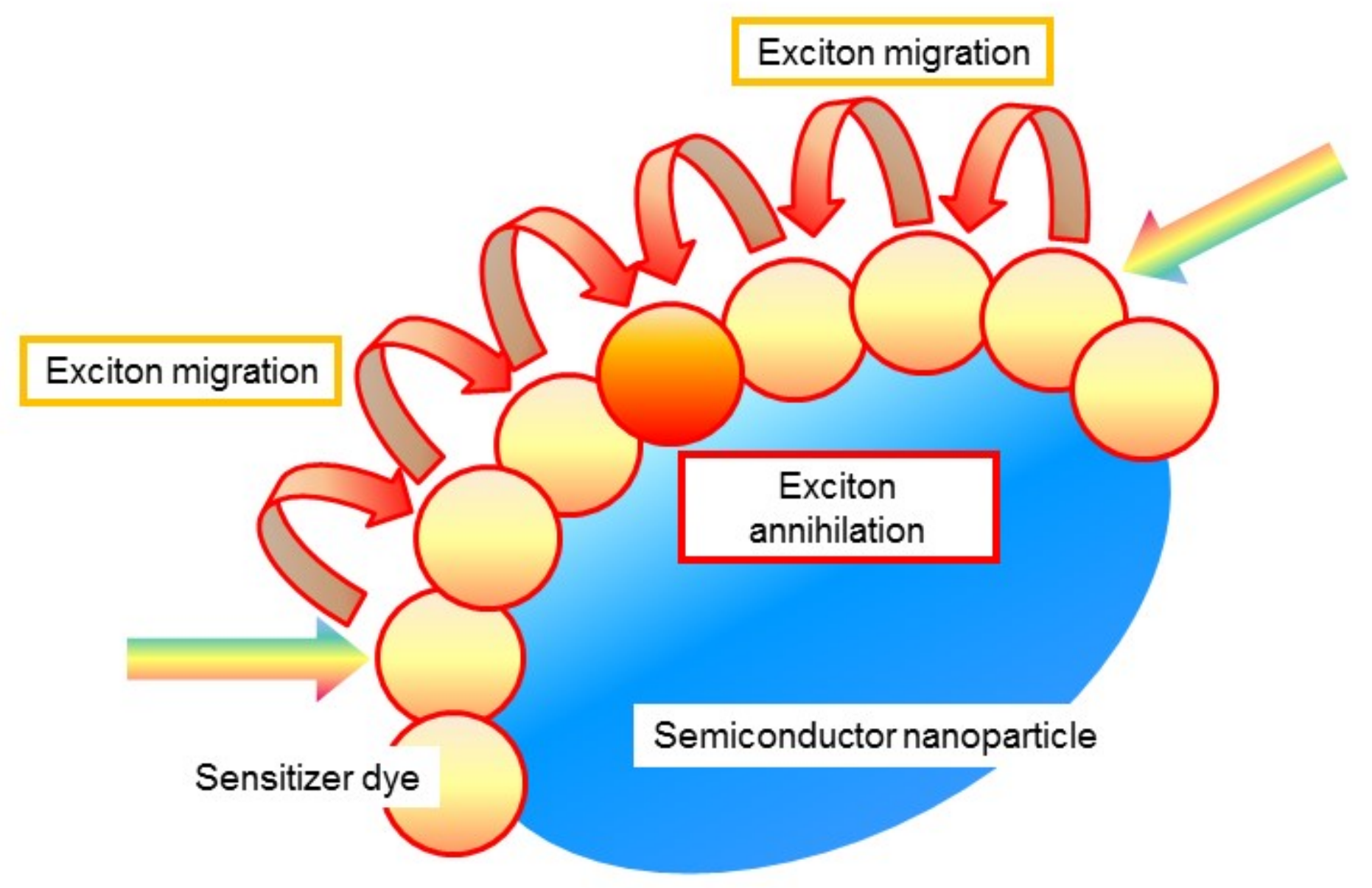

